\title{
Results Pending Completion of Investigation
}

National Cancer Institute

\section{Source}

National Cancer Institute. Results Pending Completion of Investigation. NCI Thesaurus.

Code $C 92143$.

Investigation is ongoing and results are not yet available. Do not use this code if the investigation is complete. 